\title{
UNIDADES DE HEMODINÂMICA: a produção do conhecimento ${ }^{\mathrm{a}}$
}

\author{
G raciele Fernanda da Costa $\mathrm{L} \mathrm{NCH}^{\mathrm{b}}$ \\ L aura de A zevedo G UIDO ${ }^{c}$ \\ L uiza de Oliveira PIT THAN ${ }^{d}$ \\ Juliane U M AN N e
}

\section{RESUMO}

T rata-se de uma revisão integ rativa de literatura com o objetivo de investigar o que se tem publicado sobre enfermagem em hemodinâmica nas bases de dados: Scientific E lectronic L ibrary O nline (SciE LO), M edical L iterature Analysis and R etrieval System Online (MEDLINE), Literatura Latino-A mericana e do Caribe em Ciências da Saúde (LILACS), e Base de Dados em Enfermagem (BDE NF); com os descritores: E nfermagem/ H emodinâmica e $\mathrm{N}$ ursing/ $\mathrm{H}$ emodynamics. Os dados indicam que os estudos em sua maioria for am desenvolvidos por enfermeiros, e relacionados à assistência de enfermagem. A inda, foram abordados aspectos relacionados ao reprocesso de cateteres e saúde dos profissionais de enfer magem. Contudo, pode-se perceber que a publicação de trabal hos rel acionados às unidades de hemodinâmica é escassa. N o entanto, demonstram o predomínio de pesquisas e relatos relacionados aos aspectos da assistência de enfer magem nesse setor, o que pode representar as necessidades e os problemas que permeiam o trabalho.

D escritores: H emodinâmica. Cardiologia. Cuidados de enfermagem.

\section{RESUMEN}

Se trata de una revisión integradora que tiene por objetivo investigar lo que se ha publicado sobre enfermería en hemodinámica, en las bases de datos: Scientific E lectronic L ibrary O nline (SciE L O), M edical L iterature A nalysis and R etrieval System 0 nline (M E D L IN E ), L iteratura L atinoamericana y del Caribe en Ciencias de la Salud (L IL ACS), y B ase deD atos deE nfermería (BD E N F ); con los descriptores: E nfermería/ H emodinámica y N ursing/ H emodynamics. L os datos indican que los estudios en su mayoría fueron desarrollados por enfermeros, y relacionados a la asistencia de enfermería. $F$ uer on percibidos aun, aspectos relacionados al reprocesami ento de catéteres y salud de los profesionales de enfermería. T ambién, puede perci bi rse que la publicación de trabajos relacionados a las unidades de hemodinámica es escasa. Pero, hay una demostración de la predominancia de investigaciones e informes relacionados a los aspectos de la asesoría de enfermería en ese sector, lo que puede representar las necesidades y los problemas que hay por detrás del trabajo.

D escriptores: H emodinámica. Cardiología. A tención de enfermería.

T ítulo: U nidad de hemodinamia: la producción del conocimiento.

\section{ABST RACT}

This study aimed at doing an integrative review that has as objective to investigate what has been published on nursing in hemodynamic in the following data bases: Scientific E lectronic L ibrary O nline (SciE L O), M edical L iterature A nalysis and R etrieval System 0 nline (M E D L IN E ), L atin American and Caribbean H ealth Sciences (L IL ACS), and N ursing D atabase (BDE N F); with the descriptors: E nfermagem and $\mathrm{H}$ emodinâmica and $\mathrm{N}$ ursing and $\mathrm{H}$ emodynamics. T he data indicate that the studies in his majority w ere developed by nurses, and made a list to the presence of nursing, there w ere still boarded aspects made a list to the reprocess of catheters and health of the professionals of nursing. $\mathrm{N}$ ever theless, it is noticeable that the publication of works connected with the thematic of hemodynamic is limited. H ow ever, they demonstrate the predominance of inquiries and reports making a list to the aspects of the presence of nursing in this sector, which may represent the necessities and the problems that permeate the work.

Descriptors: H emodynamics. Cardiology. N ursing care

T itle: U nit of hemodynamics: the production of the knowledge.

\footnotetext{
a Artigo originado da dissertação de M estrado apresentada em 2009 ao Programa de Pós-Graduação em E nfermagem (PPGE nf) da U niversidade Federal de Santa M aria (UF SM ), Rio G rande do Sul, Brasil.

b M estre em Enfermagem, Doutoranda em Enfermagem do PPGEnf da Universidade Federal do Rio Grande do Sul (UFRGS), Porto Alegre, Rio Grande do Sul, Brasil.

' Doutora de Enfermagem, Professora Adjunta da UFSM, Coordenadora em exercício do PPGEnf/ UF SM, Santa M aria, Rio Grande do Sul, Brasil.

d Enfermeira, E specialista em Cardiologia, M estranda do PPGEnf/ UFSM, Santa M aria, Rio Grande do Sul, Brasil.

e Enfermeira, M estranda do PPGE nf/ UFSM, Santa M aria, Rio Grande do Sul, Brasil.
} 


\section{INT RODUÇÃO}

Diante dos avanços científicos etecnológicos, têm-se as unidades de hemodinâmica (UHD) como serviços que dispõem métodos diagnósticos e ter apêuticos, por vezes mais rápidos e precisos, com técnicas eficientes visando menores riscos para o paciente.

Hemodinâmica é uma palavra originária do grego haima (sangue) e dynamis (força), significando, portanto, o estudo dos movimentos do sangue e das forças que o impulsionam ${ }^{(1)}$. São unidades que, além da cardiologia, servem de apoio para outras áreas da medicina, como neurocirurgia, radiologia, eletrofisiologia e cirurgia vascular.

A U H D apresenta-se como um campo de trabal ho rel ativamente novo para a enfermagem, sendo um serviço de alta complexidade com condições peculiares de trabalho(2). Desta maneira, a unidade de hemodinâmica é um serviço dinâmico que compr eende situações de emergências, visto os riscos aos pacientes submetidos a intervenções hemodinâmicas, com tecnologias e materiais específicos para cada procedimento ${ }^{(3,4)}$.

Q uanto ao ambiente, é uma unidade fechada, com iluminação artificial, sons e ruídos característicos dos equipamentos, e ainda emprega radiação ionizante para a real ização dos procedimentos. E m vista disso, o processo de trabal ho de enfer magem em UHD envolve riscos potenciais, dentre esses as cargas físicas, químicas, biológicas, mecânicas, mentais e emocionais ${ }^{(5)}$.

0 enfermeiro em hemodinâmica tem as responsabilidades de uma unidade com características de cuidados críticos, deve ter capacitação intelectual, ações de lider ança, atual ização e treinamento, e ainda pensamento crítico. Esse profissional deve acompanhar a evolução da tecnologia do serviço e da constante inovação de materiais ${ }^{(4,6)}$.

Diante desse contexto, o enfermeiro deve desenvolver estudos nessa área para evoluir continuamente seus conhecimentos e suas habilidades, em vista aos constantes avanços científicos, adaptações e implementações de novas tecnologias, o que tem contribuído para a complexidade dos processos de trabal ho neste setor de saúde ${ }^{(2)}$.

Contudo, a escassez de estudos na literatura brasileira sobre enfermagem em U HDs, dentre as especialidades, impulsionou-nos a desenhar este estudo, cujo objetivo foi investigar o que tem sido publicado sobre enfer magem em hemodinâmica.

\section{METODOLOGIA}

Neste estudo utilizou-se como método a revisão integrativa de literatura que tem como finalidade sintetizar resultados obtidos em pesquisas sobre um deter minado tema, de maneira sistemática e ordenada, para contribuir com o conhecimento dessa temática ${ }^{(7)}$.

Para guiar a pesquisa, formulou-se a seguinte questão: 0 que tem sido produzido na literatura sobre enfermagem em hemodinâmica?

Realizou-se a busca das publicações indexadas nas seguintes bases de dados: Scientific E lectronic $L$ ibrary 0 nline (SciE LO ), M edical L iterature A nalysis and Retrieval System Online (M EDLINE), Literatura $L$ atino-A mericana e do $C$ aribe em Ciências da Saúde (LILACS), e Base de Dados em Enfermagem (BDENF). Foram utilizados dois cruzamentos de descritores, sendo os seguintes: Enfermagem/ H emodinâmica eN ursing/ H emodynamics; justifica-se que, apesar de ambos terem o mesmo significado, apresentaram resultados diferentes quando combinados em português e inglês. D estaca-se que foi utilizado "and" entre os descritores como operador booleano.

Os critérios de inclusão foram trabalhos publicados em inglês, português e espanhol; em formato de artigos, dissertações e teses; com resumos disponíveis nas bases de dados. Como critérios de exclusão: trabalhos que abordam a assistência pediátrica em hemodinâmica; e/ ou relacionados, exclusivamente, aos estudos de parâmetros hemodinâmicos. Salienta-se que o período de publicação não foi utilizado como critério para a seleção.

A coleta de dados foi realizada em Junho de 2008, sendo que os formulários em cada base de dados foram preenchidos duas vezes com os descritores mencionados acima. Para organização da pesquisa foi elaborado um formulário para a coleta de dados composta pelos seguintes itens: autores, título, periódico, ano, método e objetivo do estudo.

Salienta-se que a busca foi real izada de forma ordenada nas bases de dados, respectivamente, SCIELO, LILACS, BDENF e por fim MEDLINE, desta maneira os itens que encontravam-se indexados em mais de uma base de dados, eram sel ecionados na primeira busca.

Posteriormente, for am lidos todos os resumos para a sel eção, os que atender am os critérios foram 
selecionados e lidos na íntegra (disponíveis online ou adquiridos pelo sistema de comutação).

A análise dos dados deu-se a partir do julgamento crítico dos dados isolados e articulados, com o intuito de caracterizar e sintetizar os estudos respondendo à questão delimitada nesta revisão integrativa de literatura(7).

D estaca-se que os dados foram inicialmente organizados a partir de freqüência absoluta (n) e percentual (\%), em seguida distribuídos pelas seguintes categorias: assistência de enfer magem a pacientes submetidos a estudos hemodinâmicos; reprocesso de cateteres; saúde dos profissionais de enfer magem em hemodinâmica.

\section{RESULT ADOS}

$\mathrm{Na}$ T abela 1, podem-se verificar os resultados encontrados a partir da busca pelos descritores, em freqüência absoluta (n) e percentual (\%) conforme cada base de dados.

D os 15 itens selecionados, 13 eram artigos e duas dissertações (uma apresentada na Escola de E nfermagem da U niversidade de São Paulo e outra na E scola N acional de Saúde Pública). Com relação à língua de publicação, apenas dois artigos foram publicados em espanhol, sendo o restante em português.

T abela 1 - Distribuição dos itens encontrados e selecionados por bases de dados. Santa M aria, RS, 2008.

\begin{tabular}{lccrr}
\hline Bases de dados & Enfermagem/ Hemodinâmica & Nursing/ Hemodynamics & \multicolumn{2}{c}{ Itens selecionados } \\
\cline { 3 - 5 } & & 2 & 4 & n \\
\hline SciELO & 4 & 14 & 7 & 46,67 \\
LILACS & 26 & 7 & 3 & 20,00 \\
BDEN F & 12 & 19 & 1 & 6,66 \\
MEDLINE & 97 & $\mathbf{4 2}$ & $\mathbf{1 5}$ & $\mathbf{1 0 0}$ \\
\hline T otal & $\mathbf{1 3 9}$ & &
\end{tabular}

Dentre os autores, apenas o artigo intitulado: "Remoção de introdutor pós-intervenção coronária percutânea: médico residente versus enfermeiro especializado"(8) foi escrito por médicos, pois os demais itens selecionados tiveram como principais autoras enfermeiras assistentes e che- fes, mestres, e ou ainda doutoras em enfermagem.

Em relação ao período das publicações dos trabal hos constatou-se que tiver am início em 1975, mas de maneira esporádica e escassa, tendo uma maior concentração a partir de 2005 (F igura 1).

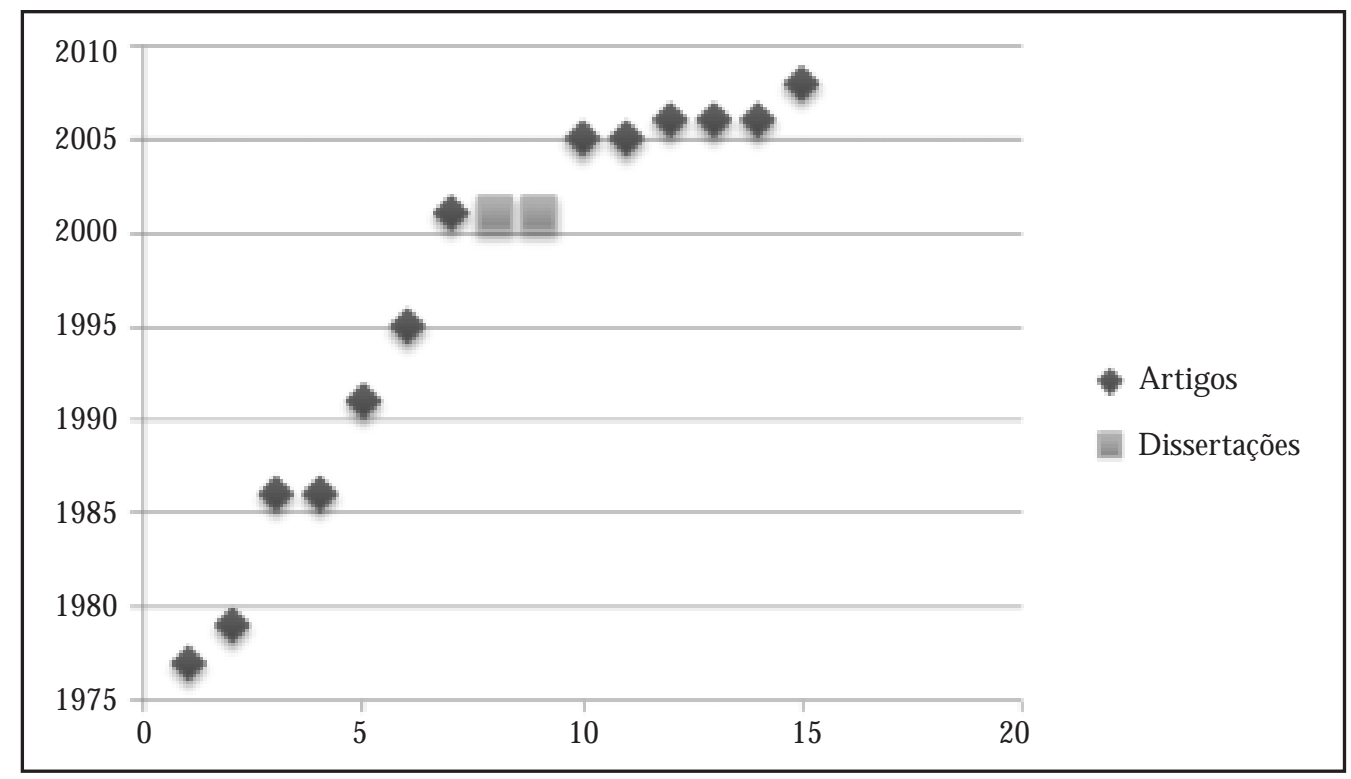

Figura 1 - Distribuição dos artigos e dissertações por período de publicação. Santa M aria, RS, 2008. 
Ainda na Figura 1, pode-se perceber que no ano de 2006 identificou-se o maior número de artigos publicados relacionados a enfermagem em hemodinâmica. No que se refere as dissertações, estas concentraram-se em 2001.

Quanto ao método utilizado nos artigos, verificou-se um total de nove classificados como artigos originais, sendo resultados de pesquisas qualitativas e quantitativas, seguido de um pequeno número de relatos de experiência e revisões de literatura (T abela 2).

T abela 2 - Distribuição dos itens de acordo com 0 método utilizado. Santa M aria, RS, 2008.

\begin{tabular}{lcc}
\hline M étodo & $\mathbf{n}$ & $\%$ \\
\hline Pesquisa qualitativa & 7 & 46,67 \\
Relato de experiência & 4 & 26,67 \\
Pesquisa quantitativa & 2 & 13,33 \\
Revisão de literatura & 2 & 13,33 \\
\hline Total & $\mathbf{1 5}$ & $\mathbf{1 0 0}$ \\
\hline
\end{tabular}

Verifica-se que as dissertações apresentaramse como pesquisas qualitativas, sendo contabilizadas, como tal, na T abela 2.

E $m$ relação à classificação das categorias dos itens, observa-se na T abela 3 um percentual maior nos que se referem a assistência de enfer magem a pacientes submetidos a estudos hemodinâmicos $(66,67 \%)$.

Tabela 3 - Itens de acordo com as categorias a que pertencem. Santa M aria, RS, 2008.

\begin{tabular}{lrc}
\hline Categoria & $\mathbf{n}$ & $\%$ \\
\hline Assistência de enfer magem a pa- & & \\
cientes submetidos a estudos & & \\
hemodinâmicos & 10 & 66,67 \\
Reprocesso de cateteres & 3 & 20,00 \\
Saúde dos profissionais de enfer- & 2 & 13,33 \\
magem em hemodinâmica & $\mathbf{1 5}$ & $\mathbf{1 0 0}$ \\
\hline Total &
\end{tabular}

É importante destacar que as dissertações pertencem a categorias difer entes, sendo que uma se refere ao reprocesso de cateter es e outra a saúde dos profissionais de enfermagem em hemodinâmica.

\section{DISCUSSÃO}

A partir desta revisão integrativa pode-se identificar que a produção relacionada à temática, enfermagem em hemodinâmica, é escassa. No entanto, percebe-se um aumento dessa produção nos últimos dez anos, o que pode estar relacionado ao aumento considerável de unidades de hemodinâmicas, e ainda a expansão dos programas da pró-graduação, assim como, o incentivo para o desenvolvimento de pesquisas na área da cardiologia.

Os dados indicam que os estudos em sua maioria foram pesquisas qualitativas, desenvolvidas por enfer meiros, e relacionados à assistência de enfermagem. E sse fato pode refletir al gumas necessidades e carências dos enfermeiros que atuam nessa área, que buscam nas pesquisas as respostas para seus questionamentos, e assim a melhoria do cuidado prestado ao paciente.

Contudo, foram abordados outros aspectos como: reprocesso de cateteres e questões relacionados à saúde dos profissionais de enfermagem, sendo assim a discussão será apresentada de acordo com as categorias encontradas a partir da semelhança entre os temas.

\section{Assistência de enfermagem a pacientes submetidos a estudos hemodinâmicos}

Essa categoria foi composta por dez artigos, os quais apresentam a atuação da enfermagem em hemodinâmica, relatam algumas questões específicas desta unidade, e ainda destacam a importância do cuidado a estes pacientes.

Alguns autores convergem, em seus relatos, sobre o papel do enfermeio nos estudos hemodinâmicos, sendo que este desenvolve suas atividades assistênciais em três momentos (pré, trans e pós procedimento) $)^{(1,3,9)}$.

A orientação, a avaliação e o preparo tanto físico quanto emocional (pscicológico) aos pacientes fazem parte do período pré-procedimento. $N$ este momento, o conhecimento dos temores, dúvidas e expectativas dos pacientes em relação aos exames torna-se fundamental para que o enfermeiro possa assistí-lo de maneira individual izada ${ }^{(1,6,10)}$

N os estudos hemodinâmicos são utilizadas substâncias radiopacas, adequadas para injeção na circulação como agente de contraste radiopaco, para produzir radiopacicidade diagnóstica. N o entan- 
to, esse contraste pode desencadear reações que podem variar desde reações mínimas até choque anafilático. Contudo, para evitar as possíveis complicações, o enfermeiro deve investigar história de reações de sensibilidade e alérgicas, de hipertireoidismo, insuficiência renal ou qualquer outra alterção orgânica ${ }^{(9,11)}$. Por fim deve orientar o paciente sobre os efeitos colaterais do contraste para que o mesmo possa comunicar a equipe e esta possa intervir imediatamente $e^{(3,9)}$.

Durante 0 exame, 0 enfermeiro deve estar atento ao traçado eletrocardiográfico e suas alterações, a possibilidade de administração de medicações, bem como atentar para sinais ou sintomas sugestivos de complicações ${ }^{(3)}$.

A monitorização hemodinâmica é realizada por um sistema operacional especifico para estudos hemodinâmicos, o que possibilita análise cardiológica dos pacientes durante a realização do estudo ${ }^{(12)}$. Com esse, o enfermeiro pode verificar os parâmetros hemodinâmicos, e detectar alterações que possam ocorrer durante o exame.

A pós o procedimento, é retirado o introdutor arterial, observado possível sang ramento, e ainda, realizados curativos necessários, controle dos sinais vitais, e o encaminhamento para a sala de recuper ação do serviço ou unidade de inter nação(1,3,9).

A remoção do introdutor arterial pós-intervenção cor onária percutânea pode ser realizada pelo enfermeiro desde que o mesmo tenha E specialização em T erapia I ntensiva ou em U nidade de Hemodinâmica, segundo o Parecer T écnico do Conselho Regional de Enfermagem do Distrito Federal no 014/2001(13). No Brasil, foi realizado o estudo Remoção de Introdutor A rterial Pós-intervenções Coronárias Percutâneas: médico residente versus enfermeiro (RIPRE), sendo que o mesmo concluiu que a retirada de introdutor arterial pode ser realizada com segurança e sem maiores complicações tanto pelo enfer meiro especial ista quanto pelo médico residente ${ }^{(8)}$. Salienta-se, ainda, a importância do treinamento para estes profissionais a fim de obter assistência qual ificada.

Dentre os aspectos que influenciam a assistência de enfermagem, pode-se considerar a influência da terceirização no processo de cuidado de enfermagem aos pacientes submetidos a estudos hemodinâmicos e possíveis conflitos. Destaca-se dificuldades de integ ração e continuidade dos cuidados entre serviços terceirizados e não terceirizados, ainda evidencia-se a necessidade de uma equipe integrada entre o serviços de hemodinâmicas e outras unidades de internações ${ }^{(14)}$.

Possivelmente os conflitos ocorrem nos momentos de admissão ou encaminhamento dos pacientes para as unidades de internação ou cuidados intensivos. $\mathrm{Ou}$, ainda, com unidades de apoio, geralmente responsáveis pela dieta dos pacientes, pela limpeza das unidades e pela esterilização dos artigos médico-hospitalares. Evidencia-se que o processo de trabal ho de enfer magem em U HD se dará de forma efetiva a partir do apoio de outras unidades e profissionais. Assim, o enfermeiro deve gerenciar da melhor maneira possível os conflitos com outras unidades, vista a importância de cada um nesse processo, dessa maneira, evitando eventuais situações que possam ser consideradas estressantes.

O utro estudo realizado por enfermeiras propõe a classificação de pacientes quanto a necessidade de cuidados, sendo que estes podem apresentar, após a inter veção, diferentes graus de cuidados. Segundo as autoras, a classificação pode trazer subsídios para gar antir uma assistência de qualidade no redimencionamento da equipe de enfermagem, proporcionando maior satisfação, rendimento, e mais eficiência ao trabal ho, além de menor tempo de internação ${ }^{(15)}$.

A atuação do enfermeiro em unidade de hemodinâmica exige conhecimento complexo, desde tarefas simples até as que exijam uma formação técnico-cientifíca adequada e específica. A inda, para que esse profissional desempenhe suas funções com ver satilidade e qualidade da assistência ao paciente, o mesmo necessita de experiência, atualizações constantes, e desenvolvimento de conhecimentos e habilidades específicas para área.

\section{Reprocesso de cateteres}

Para a realização dos procedimentos em hemodinâmica e qual idade dos serviços, cada vez mais se desenvolvem materiais com tecnologia e qualidade. Paralelo a isso, a área de H emodinâmica mantém o reprocesso de uma quantidade significativa de materiais, tendo em vista 0 al to custo das intervenções percutâneas, o que tem sido acompanhado com preocupação, especialmente, em relação aos eventos adversos decorrentes desse reprocesso.

E m vista disto, alguns autores em busca de respostas e evidências em relação a questões referentes aos efeitos, eficácia e riscos do reprocesso 
dos cateteres, realizar am uma revisão na literatura científica(16). E ainda, destacaram três preocupações relacionadas ao reuso dos artigos médicos de uso único: a eficácia do processo e a integridade desses materiais; a segurança dos profissionais envolvidos; e principalmente, os riscos de contaminações destes pacientes ${ }^{(17)}$.

Os artigos análisados na referida revisão apontam como principal problema o reuso de materiais de uso único, sendo que essa atitude perpassa por aspectos econômicos relacionados aos custos que envolvem o número de reprocessos, o produto utilizado na limpeza, dentre outros. No entanto, por vezes, a estratégia para controlar custos dos procedimentos acaba prejudicando a qual idade do processo, e consequentemente, expondo pacientes e profissionais a riscos.

Estudos identificaram e analisaram aspectos relacionados a decisão de reprocessar e reutilizar cateteres originalmente feitos para uso único ${ }^{(17,18)}$. D entre esses, constatou que 0 aspecto econômico é a principal causa da reutilização e que a responsabilidade por esta decisão não é conhecida por um único profissional (médico ou enfermeiro), mas por vezes tem como responsável a instituição.

E $m$ vista disso, recentemente, a A gência $\mathrm{N}$ acional de Vigilância Sanitária (AN VISA) publicou a Resolução 2.606 que dispõe sobre as diretrizes para elaboração, validação e implementação de protocolos de reprocessamento de produtos médicoshospitalares e dá outras providências ${ }^{(19)}$. E ssa regulamentação da atividade visa a segurança dos pacientes e profissionais, e a eficácia do reprocessamento destes materiais.

\section{Saúde dos profissionais de enfermagem em hemodinâmica}

Os dois estudos dessa categoria apresentam questões referentes à saúde dos profissionais de enfer magem em hemodinâmica, sendo investigadas as condições de trabal ho, riscos ocupacionais, e principalmente, relativos à peculiaridade da radiação ionizante.

U m estudo apresenta o processo de trabalho de enfermagem em hemodinâmica relacionado às cargas de trabal ho e fatores de riscos à saúde do trabalhador ${ }^{(5)}$. Caracteriza esse setor como alta complexidade, elevado nível tecnológico, atividades excessivas evariadas, ambiente estressante pela exigência da dinâmica das ações, e ritmo de produ- ção intenso, fatores que impõem a sobrecarga de trabal ho para a equipe de enfer magem.

Ainda, esse estudo possibilitou identificar alguns problemas que colocam em risco à saúde, segurança e conforto desses trabalhadores. Dentre os principais, está o risco de contaminação com microorganismos e acidentes biológicos devido à intensa manipulação de instrumentos perfuro-cortantes, as cargas emocionais e mentais decorrentes da exigência de conhecimentos e habilidades específicas, da tomada de decisão, da assistência e gerência do cuidado, e por fim destaca os riscos ambientais como o barulho, a iluminação, o desconforto térmico e a exposição ao risco de radiação, uma vez que esse é inerente ao processo de trabalho(5).

E $m$ relação às atividades que envolvem radiação ionizante real izadas por profissionais de enfermagem, outro estudo teve como objetivo verificar o cumprimento da Resolução 211/ 98 do Conselho Federal de Enfermagem (COFEn) que dispõe sobre a atuação desses profissionais em serviços de radiodiagnóstico na área da saúde ${ }^{(20)}$. No entanto, demonstrou que os setores das instituições pesquisadas não cumprem a referida Resolução na sua totalidade, e ainda os sujeitos informam desconhecê-la, não recebendo capacitação dirigida aos cuidados mínimos de proteção radiológica, assim expondo-se a riscos desse agente físico(21).

Os profissionais de enfermagem que atuam em unidade de hemodinâmica estão expostos aos riscos inerentes ao processo de trabal ho em unidades fechadas e de alta complexidade. No entanto, tem 0 agravante posto pela radiação ionizante, 0 que exige medidas preventivas e um controle rigoroso da saúde destes profissionais.

\section{CONSIDERAÇÕES FINAIS}

Com a real ização deste estudo fica evidente a escassez de trabalhos relacionados às unidades de hemodinâmica. N o entanto, as publicações apresentadas demonstram o predomínio de pesquisas e relatos relacionados aos aspectos da assistência de enfermagem nesse setor. E sse predomínio pode representar as necessidades e problemas que permeiam o seu trabalho.

Outra questão relacionada ao processo de trabalho em hemodinâmica é a tomada de decisão quanto ao reprocesso e reutilização de cateter es de 
uso único. Contudo, essa decisão envolve diferentes aspectos, que por vezes podem ocasionar conflitos e questionamentos para os profissionais, sendo pressionados pela instituição ou por seus supervisores, por condutas exigidas pela ANVISA, eainda a pela de segurança aos pacientes.

No que se refere aos profissionais de enfermagem, os riscos a saúde têm sido minimamente abordados nos estudos. No entanto, estes trabal hadores pelas especificidades do processo de trabaIho, estão expostos aos diferentes riscos, como 0 biológico, químico, e principalmente ao radiológico. Assim, em unidade de hemodinâmica esses são el ementos for temente definidos como agentes causais de adoecimento no trabal ho devido às características e complexidade do setor.

D iante desta revisão integ rativa sugere-se que o desenvolvimento de estudos relacionados a esta temática devem ser incentivados, aprofundados e divulgados, para que contribuam com o desenvolvimento de outras pesquisas, ou ainda, que auxiliem a atuação e a tomada de decisão dos enfer meiros em U nidades de H emodinâmica.

\section{REFERÊ NCIAS}

1 Daubermann LV, Silva N P. Papel do enfermeiro nos estudos hemodinâmicos. Rev G aúcha Enferm. 1986; 7(1):13-22

2 Linch GFC. E stresse de enfermeiros em unidade de hemodinâmica [ dissertação] . Santa M aria: U niversidade Federal de Santa M aria; 2009.

3 G onçalves JD M, Barbosa PM K, Brazoloto V, Shiotsu $\mathrm{CH}$. A s atividades assistenciais do enfer meiro em unidade de hemodinâmica. A cta Paul E nferm. 1991;4(1): 48-54.

4 Cunha A IG, Santos JF V, Bal bieris VC, Silva EV. A enfermagem na cardiologia invasiva. São Paulo: A theneu; 2007.

5 Santos PR. E studo do processo de trabal ho da enfermagem em hemodinâmica: desgastes, cargas de trabalho e fatores de riscos à saúde do trabal hador [ dissertação] . Rio de Janeiro: E scola $N$ acional de Saúde Pública, F undação O svaldo Cruz; 2001.

6 Betelu AE, M ontes YJ, Artazcoz MAA, G oñi EM, Sarnago M T S. Cateterismo cardíaco: actuación de enfermería en una unidad de críticos. Enferm Intensiva. 2006;17(1):3-11.
7 Roman $A R$, F riedlander M R. Revisão integrativa de pesquisa aplicada à enfermagem. Cogitare E nferm. 1998;3(2):109-12.

8 Solano JDC, M eireles GCX, A breu L M , Forte AAC, Sumita M K, H ayaschi J H. Remoção de introdutor arterial pós-intervenção coronária percutânea: médico residente versus enfermeiro especializado.J Vasc Bras. 2006;5(1):42-6

9 L eite J L, Barbosa NA. A atuação da enfermagem no estudo hemodinâmico. E nferm N ov Dimen. 1977;3(3): 147-53.

10 Boudet Sani M M, Robert Despaigne M. Resultado del trabajo de la enfermera en la sección de hemodinámica intervencionista. Santiago de Cuba: [ s.n.] ; 1995.

11 Ernesto DZL, Oliveira M ED, Zanei SSV. A gentes contrastantes no estudo hemodinâmico: diretrizes para a enfermagem. Rev Paul E nferm. 1986;6(3):1258.

12 Silva J. Computação em hemodinâmica: aspectos de enfer magem. E nferm N ov D imen. 1979;5(1):37-40.

13 Conselho Regional de Enfermagem (DF). Parecer técnico no 014, de 12 de novembro de 2001: competência legal do profissional enfermeiro na retirada de cateter introdutor após procedimentos de natureza hemodinâmica [ I nter net] . Brasília (DF); 2001 [ citado 2009 out 12] . Disponível em: http:/ / www. coren-df.org.br/ portal $/$ index.php?option $=$ com content $\&$ view $=$ article $\& i d=239$ : parecer - tecnico-no0142001\&catid=38: pareceres\&l temid $=73$.

14 Zamberlan C, Siqueira H CH . A terceirização nos serviços e conseqüências no cuidar em enfermagem. Rev Bras Enferm. 2005;58(6):727-30.

$15 \mathrm{H}$ ammermüller A, Rabelo E R, Goldmeier S, Azzolin KO. Classificação de pacientes atendidos em uma unidade de hemodinâmica segundo o grau de dependência dos cuidados de enfermagem. A cta Paul E nferm. 2008:21(1):72-6.

16 Ribeiro SM CP, G raziano KY, A Ifa M M , G oveia VR. Reprocessamento de cateteres cardíacos: uma revisão. Braz J Cardiovasc Surg. 2006;21(3):334-42.

17 Baffi SH O. Reprocessamento e reutilizaçäo de cateteres de hemodinâmica: a busca da qualidade nesta prática [ dissertação] . São Paulo: Escola de Enfermagem, U niversidade de São Paulo; 2001. 
18 Baffi SH, Lacerda RA. A busca de qualidade no reprocessamento e na reutilização de cateteres de hemodinâmica. Rev SO BE CC. 2001;5(6):23-30.

19 M inistério da Saúde (BR ), A gência N acional de Vigilância Sanitária. Resolução n⒉606, de 11 de agosto de 2006: dispõe sobre as diretrizes para el abor ação, validação e implantação de protocolos de reprocessamento de produtos médicos e dá outras providências [ I nternet] . Brasília (D F ); 2006 [ citado 2009 out 12] . D isponível em: http:/ / www.anvisa.gov.br/ legis/ resol/ 2006/ re/2606_06re.htm.
20 Consel ho Federal de Enfermagem (BR). Resolução no 211, de 01 de julho de 1998: dispõe sobre a atuação dos profissionais de enfer magem que trabal ham com radiação ionizante [ Internet] . Brasília (DF); 2004 [ citado 2009 out 12] . Disponível em: http:// www.portal cofen.g ov.br/ 2007/ materias.asp? A rticlel $D=7046 \&$ sectionl $D=34$.

21 Flor RC, Kirchhof ALC. R adiação ionizante e o cumprimento de resolução do Conselho Federal de Enfermagem. Rev Enferm UERJ. 2005;13(3):34753.

Recebido em: 29/ 09/2009

A provado em: 21/ 12/ 2009

\section{Endereço da autora / Dirección del autor /} Author's address:

L aura de A zevedo G uido

Av. Roraima, 1000, Prédio 16-CCS, Sala 1302,

Bairro Camobi

97105-900, Santa M aria, RS

E-mail:Lauraazevedoguido@gmail.com 